\title{
Investigating the circulatory-respiratory response of elderly people during Tai Chi Yuttari-exercise
}

\author{
Takuo Nomura ${ }^{1,2^{*}}$, Yoshiteru Akezaki ${ }^{1,2}$, Kohei Mori ${ }^{1,2}$, Emi Nakamata $^{1,2}$, Fuminari Asada ${ }^{3}$, \\ Yoshiaki Mori ${ }^{1}$, Satoru Kai ${ }^{1,2}$, Masahito Watanabe ${ }^{1}$ \\ ${ }^{1}$ Department of Rehabilitation Sciences, Kansai University of Welfare Sciences, Osaka, Japan; \\ *Corresponding Author: tnomura@,fuksi-kagk-ac.jp \\ ${ }^{2}$ Theraputic Exercise Research Laboratory, Kansai University of Welfare Sciences, Osaka, Japan \\ ${ }^{3}$ Osaka Rosai Hospital Center for Preventive Medicine, Osaka, Japan
}

Received 10 October 2013; revised 15 November 2013; accepted 25 November 2013

Copyright (C) 2013 Takuo Nomura et al. This is an open access article distributed under the Creative Commons Attribution License, which permits unrestricted use, distribution, and reproduction in any medium, provided the original work is properly cited.

\section{ABSTRACT}

The purpose of this study was to examine the circulatory-respiratory dynamics in elderly people during Tai Chi Yuttari-exercise. The subjects were females with an average age of 74 years who had been continuously practicing Tai Chi Yuttari-exercise for at least six months. The heart rate and blood pressure were measured before and after the subjects performed four different versions of Tai Chi Yuttari-exercise. The perceived exertion was evaluated using the Borg scale. The respiratory rate, oxygen uptake and respiratory quotient (RQ) were measured using an expired gas analyzer. The results showed an increase in heart rate during exercise, but confirmed that the rise in HR was below the safety limit. The blood pressure tended to be low post-exercise. Regarding perceived exertion, the maximum heart-lungs Borg scale response was 9 (very light). A slight increase in the respiratory rate was observed while exercising, but no abnormal breathing patterns were observed. The average metabolic equivalents (METs) of the exercise ranged from 1.34 to 1.87 , and the average $R Q$ ranged from 0.82 to 0.90 . The circulatory-respiratory dynamics observed in this study verified that elderly females with an average age of $\mathbf{7 0}$ years could safely perform the Tai Chi Yuttari-exercise. This study helped obtain basic data for expanding the indications for this exercise.

Keywords: Tai Chi Chuan; Exercise Therapy; Aged; Circulatory and Respiratory Physiological Phenomena; Rehabilitation

\section{INTRODUCTION}

Tai Chi Chuan is an ancient Chinese martial art that does not require any special equipment or setting. Tai Chi Chuan activates the whole body using balanced movements without causing undue stress. It has been shown to improve physical and psychological functions, enhance balance, reduce the rate of falling and improve cardiorespiratory functions after coronary artery bypass surgery [1-3]. Tai Chi Chuan has achieved global recognition as an exercise on par with Yoga and Pilates, although because of the stress that the exercise exerts on the knees and because it demands maximal muscular strength [4], adaptations for the elderly are needed.

For this reason, Kitakata City in Fukushima Prefecture, Fukushima Medical University, and others jointly developed a modified exercise (Tai Chi Yuttari-exercise) that incorporated Tai Chi Chuan that could be performed safely and easily by the elderly and could be practiced alone and for long periods. Continuous practice of Tai Chi Yuttari-exercise has been shown to increase physical functions and abilities such as balance [5,6], to improve the vascular function [7] and help prevent the need for long-term care [8]. In past sessions of Tai Chi Yuttariexercise conducted in our exercise classroom, we did not observe any risk events involving the respiratory or circulatory systems, confirming that the elderly could safely perform this exercise. However, the circulatory-respiratory dynamics during Tai Chi Yuttari-exercise has not yet been examined in detail. Before this specific exercise is adapted to additional patient populations, such as those with cardiovascular diseases, obtaining basic data regarding its effects on the circulatory-respiratory dynamics is needed. This study evaluated the circulatory-respiratory dynamics of elderly people aged at least 70 years before, during, and after Tai Chi Yuttari-exercise to obtain 
basic data on the circulatory-respiratory dynamics during this type of exercise.

\section{METHODS}

\subsection{Summary of Tai Chi Yuttari-Exercise}

Figure 1 shows typical pictures of Tai Chi Yuttari-exercise. Tai Chi Yuttari-exercise is composed of four different versions: two sitting-position patterns (basic version, $12 \mathrm{~min}$; advanced version, $7 \mathrm{~min}$ ) and two standing-position patterns (basic version, $14 \mathrm{~min}$; advanced version, $8.5 \mathrm{~min}$ ) [6]. All movements in the sitting-position patterns are performed while sitting, and a chair or other object is placed nearby during the standing-position patterns to prevent falls. The exercise involves slow movements and does not require rapid movements. All versions are characterized by beginning with slow movements and ending with a deep breath. In addition, the exercise is simple enough to be learned by watching it on a DVD [9] or looking at an easy-to-understand pamphlet. There is no need for a professional instructor.

\subsection{Subjects}

The subjects included four females with an average age of 74 years who were participating in a clinical study entitled "Effect of Tai Chi Yuttari-exercise program on preventing conditions that require long-term care in community-dwelling elderly people" (UMIN000006991) that was initiated in January 2012 (Table 1). The muscle mass of the participants, as measured using a bodycomposition analyzer (InBody S10, Biospace, Seoul, Korea), was within the reference range. Two subjects had earlier established an exercise habit of at least two sessions per week, consisting of at least $30 \mathrm{~min}$ per session, for at least one year. The other two subjects had never previously participated in this exercise. One subject had experienced a fall in the previous year. All of the subjects had and were being treated for hypertension or dyslipidemia, but none were under any exercise restrictions.

The data used to verify the respiratory and circulatory system safety were measured in October and November 2012, a time when all the subjects had been practicing the four versions of the exercise for at least six months. The study was explained to the subjects in writing and orally, and each subject provided their written, as well as oral, consent for participation. The research ethics committee of Kansai University of Welfare Sciences approved this study.

\subsection{Procedure, Evaluation Items and Analysis}

Two of the four exercises were randomly selected, and data were collected at 30-min intervals. Data on the re- maining two exercises were measured after at least one day had passed between exercise sessions. The items evaluated were as follows: blood pressure while resting in the sitting position and within 2 min of finishing the exercise; perceived exertion of the heart-lungs and lower limbs (Borg scale) [10] before and after exercise; oxygen uptake $\left(\mathrm{VO}_{2}\right)$, respiratory quotient $(\mathrm{RQ})$, the respiration rate (RR) measured using a portable expired gas monitor (Aerosonic AT-1100, ANIMA Corp., Tokyo, Japan), and the heart rate (HR) measured using a HR monitor (POLAR S610i, POLAR Electro, Kempele, Finland). The differences in blood pressure before and after exercise were averaged. The data on expired gases were collected when the subjects were fully at rest. Measurements were taken for $3 \mathrm{~min}$ in the sitting position, as well as while exercising. Data recorded using the breath-by-breath method was averaged for the $3 \mathrm{~min}$ of rest and for every 1 min during the exercise. The $\mathrm{VO}_{2}$ was calculated as metabolic equivalents (METs) using the resting value as the baseline. Data analyses were performed using the IBM SPSS statistics software program (version 19).

\section{RESULTS}

Figure 2 shows a graph of the four subjects' average HR and RR while performing the different versions. The HR increased during all versions, but the rise was largest in the advanced version standing-position pattern. The maximum HR in the sitting-position patterns was 91 beats/min and in standing-position patterns, it was 103 beats/min, although the $\mathrm{k}$ value was less than 0.5 when using the Karvonen formula [11]. None of the subjects exhibited a marked rise in blood pressure after the exercise, but compared to before exercise, the systolic blood pressure in particular showed a decreasing trend postexercise (Table 2). Regarding perceived exertion, the maximum heart-lungs Borg scale responses ranged from 7 (very, very light) to 9 (very light) for all four subjects in both the sitting-position and standing-position patterns. For the lower limbs, the response for one subject was 13 (somewhat hard) regarding a standing-position pattern, but the responses of the other three subjects ranged from 9 (very light) to 11 (fairly light).

The RR slightly increased during exercise, but no abnormal breathing patterns, such as tachypnea and polypnea, were observed in any of the subjects. The average METs for one of the subjects was $3.62 \mathrm{~mL} \mathrm{O}_{2}$ per $\mathrm{kg} / \mathrm{min}$. The average METs for the sitting-position and standing-position patterns was $1.34-1.87$, and the average RQ ranged from 0.82 to 0.90 .

\section{DISCUSSION}

Although a large number of exercises that were developed to prevent the need for long-term care also con- 

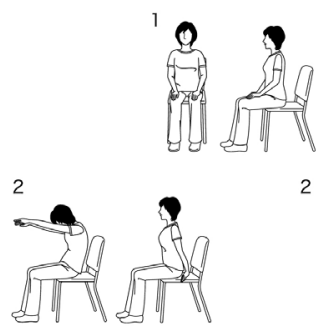

3
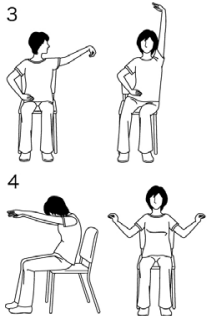

5
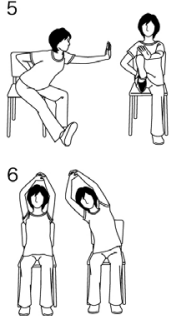

7
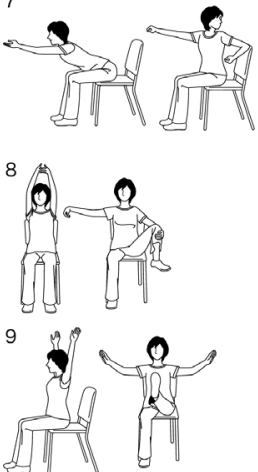

10

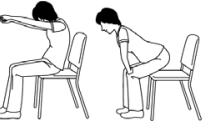

11
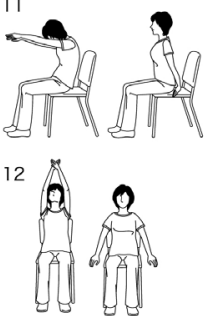

Basic version Sitting-position pattern

3
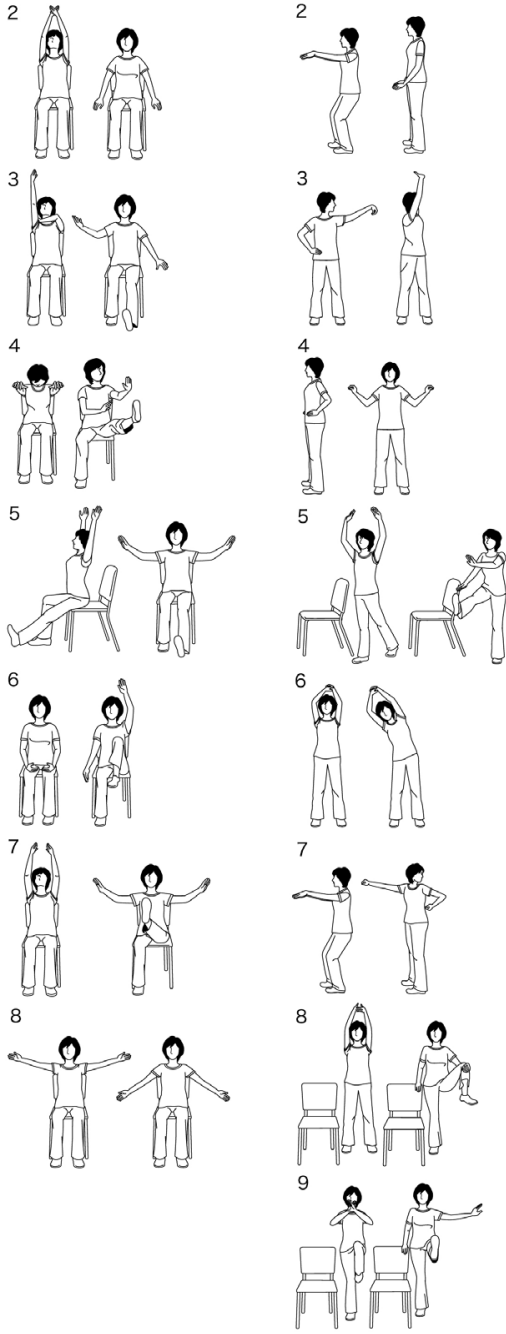

10

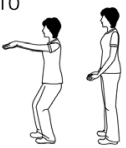

11
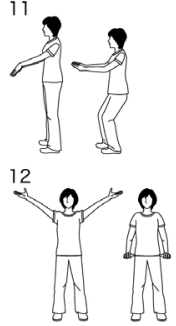

Basic version Standing-position pattern

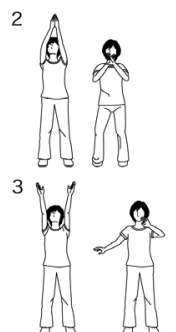

4
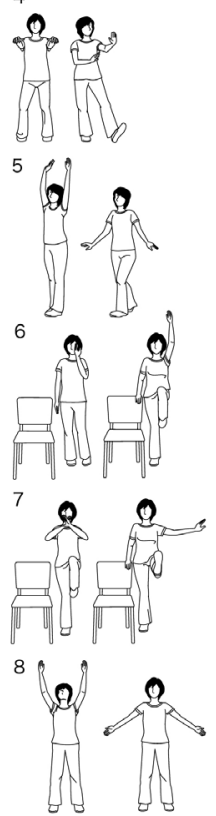

Figure 1. Typical pictures of Tai Chi Yuttari-exercise. Sitting-position pattern (left side) and standing-position pattern (right side), each the basic version of 12 patterns and the advanced version of 8 patterns. 


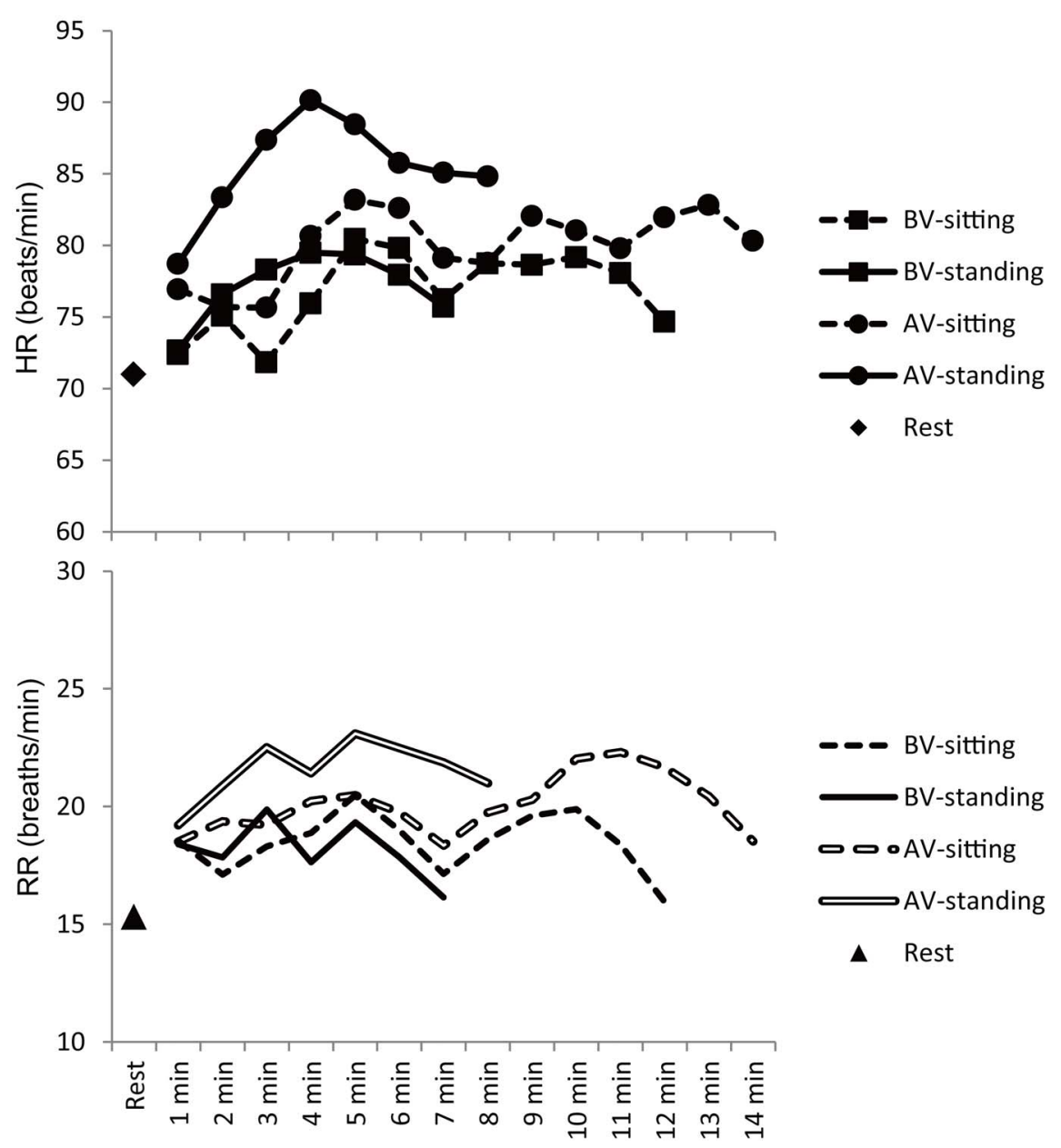

Figure 2. Average heart rates (upper figure) and respiratory rates (lower figure) during Tai Chi Yuttari-exercise. BV-sitting, basic version sitting-position pattern; BV-standing, basic version standing-position pattern; AV-sitting, advanced version sitting-position pattern; AV-standing, advanced version standing-position pattern.

Table 1. The clinical characteristics of the study subjects.

\begin{tabular}{lccccccccccc}
\hline & $\begin{array}{c}\text { Age } \\
(\mathrm{yr})\end{array}$ & $\begin{array}{c}\text { Height } \\
(\mathrm{cm})\end{array}$ & $\begin{array}{c}\text { BW } \\
(\mathrm{kg})\end{array}$ & $\begin{array}{c}\text { MV } \\
(\mathrm{kg})\end{array}$ & $\begin{array}{c}\text { SFV } \\
(\mathrm{kg})\end{array}$ & $\begin{array}{c}\text { SBP } \\
(\mathrm{mmHg})\end{array}$ & $\begin{array}{c}\text { DBP } \\
(\mathrm{mmHg})\end{array}$ & $\begin{array}{c}\text { HR } \\
(\mathrm{b} / \mathrm{min})\end{array}$ & $\begin{array}{c}\text { Exercise } \\
\text { habit }\end{array}$ & $\begin{array}{c}\text { History of } \\
\text { falls }\end{array}$ & $\begin{array}{c}\text { Chronic } \\
\text { disease }\end{array}$ \\
\hline Case 1 & 72 & 152.0 & 62.0 & 35.7 & 24.2 & 136 & 85 & 71 & + & - & HT \\
Case 2 & 75 & 149.0 & 48.0 & 32.5 & 13.7 & 154 & 83 & 74 & - & + & HT \\
Case 3 & 74 & 145.5 & 50.9 & 31.4 & 17.7 & 144 & 89 & 72 & + & - & DL \\
Case 4 & 75 & 157.0 & 54.9 & 34.5 & 18.4 & 119 & 76 & 69 & - & - & HT, DL \\
\hline
\end{tabular}

Abbreviations: BW, body weight; MV, muscle volume; SFV, somatic fat volume; SBP, systolic blood pressure; DBP, diastolic blood pressure; HT, hyper tension; DL, dyslipidemia.

Table 2. BP variation, average METs and RQ for Tai Chi Yuttari-exercise.

\begin{tabular}{ccccc}
\hline & \multicolumn{2}{c}{ Sitting-position pattern } & \multicolumn{2}{c}{ Standing-position pattern } \\
& Basic version & Advanced version & Basic version & Advanced version \\
\hline SBP variation (mmHg) & $-5.6 \pm 10.7$ & $-9.6 \pm 13.2$ & $-0.2 \pm 4.5$ & $-6.2 \pm 9.1$ \\
DBP variation (mmHg) & $2.8 \pm 5.0$ & $-3.6 \pm 3.6$ & $0.4 \pm 2.2$ & $1.4 \pm 4.8$ \\
Average METs & $1.51 \pm 0.21$ & $1.34 \pm 0.10$ & $1.80 \pm 0.29$ & $1.87 \pm 0.25$ \\
Average RQ & $0.85 \pm 0.02$ & $0.90 \pm 0.03$ & $0.82 \pm 0.01$ & $0.86 \pm 0.03$ \\
\hline
\end{tabular}

Data are the mean \pm SD. Abbreviations: SBP, systolic blood pressure; DBP, diastolic blood pressure; METs, metabolic equivalents; RQ, respiratory quotient. 
sider regional characteristics of the participants and typical exercise activities, it is important to scientifically verify their safety and effectiveness, as well as consider how to adapt these to different subjects. This study examined the Tai Chi Yuttari-exercise, for which much evidence has been established regarding elderly people [5-8]. The objective of the present study was to evaluate the circulatory-respiratory dynamics during the exercise and to obtain basic data in order to adapt it to other patient populations, such as those in need of rehabilitation.

First, although the HR increased from rest by a maximum average of 20 beats/min during the advanced version standing-position pattern, the maximum increase during the other versions was an average of 15 beats $/ \mathrm{min}$, indicating that the rise in the HR during the advanced version standing-position pattern was the largest among the four versions of the exercise. The heart-lungs Borg scale responses for the advanced version standing-position pattern ranged from 7 (very, very light) to 9 (very light), which were similar to those for the other versions. In addition, the value of $\mathrm{k}$ was under 0.5 when using the Karvonen formula for the HR increase, confirming that the rise in the HR was below the safety limit. During the Arakawa Koroban-exercise, which has recently been adapted for use by elderly people to prevent the need for long-term care, and the Arakawa Theraban-exercise, which was developed for frail elderly people, the maximum HR increases to 98 beats $/ \mathrm{min}$ and 82 beats $/ \mathrm{min}$, respectively [12]. During the Tai Chi Yuttari-exercise, the maximum HR for the sitting-position patterns was 91 beats/min, and that for the standing-position patterns was 103 beats $/ \mathrm{min}$. These findings suggest that this exercise is similar in intensity to exercises that are commonly used to prevent the need for long-term care.

Next, a tendency toward reduced blood pressure was observed after exercise. This may be attributable to the deep breaths taken during the cooling down phase in all Tai Chi Yuttari-exercise versions, and is considered to be a characteristic of this exercise. The expired gas analyses showed a slight increase in the RR during exercise, but none of the subjects exhibited an abnormal breathing pattern. This shows that respiration at a fixed rhythm can be maintained during the continuous movements of Tai Chi Yuttari-exercise. It also indicates that this exercise can be adapted for patients prohibited from performing Valsalva-type movements, such as patients with diabetic retinopathy. One limitation of this study was that the blood pressure could not be monitored during the exercises. However, because the maximum heart-lungs Borg's scale response was 9 (very light) and the breathing patterns were regular during the exercises, it may be possible that sudden increases in blood pressure did not occur. The subjects evaluated in this study consisted of only four females with an average age of 74 years, and further studies are needed to determine whether similar findings are true for males, those with poorer physical function, those who are more than 75 years old, and those with the presence of a disease. However, our present data suggest that Tai Chi Yuttari-exercise is safe for elderly subjects and may improve their circulatory-respiratory dynamics.

When developing exercises that take into account regional and cultural characteristics, it is necessary to think about their scope of application by fully grasping their effects on the body. Some exercises have been developed as high-intensity activities, with the maximum METs exceeding 7.0 [13]. Therefore, in addition to safety limits, the intensity and effectiveness of the exercises should be considered. The Japanese Ministry of Health, Labour and Welfare's "Exercise Guide 2006," [14] which was aimed at maintaining and improving health and preventing lifestyle-related diseases, recommends physical activity and exercises of moderate intensity, with METs at 3.0 or above. The average METs of the Tai Chi Yuttari-exercise ranged from 1.34 to 1.87 , and the average $\mathrm{RQ}$ ranged from 0.82 to 0.90 . The RQ values indicate that the intensity of this exercise influences lipid metabolism and reduces obesity, although the METs values were lower than those recommended by the Ministry of Health, Labour and Welfare. An intervention study on middle-aged and elderly females [15] suggested that low-intensity exercise could improve arterial distensibility. Therefore, even low-intensity exercise interventions for elderly subjects can be expected to have a certain effect on lowering the risk for lifestyle-related diseases, such as by changing the degree of arterial distensibility.

\section{CONCLUSION}

Among the different types of Tai Chi Yuttari-exercise, the advanced version standing-position pattern had the largest effect on the circulatory and respiratory systems, although this was below the safety limit. A characteristic of this exercise is that it does not significantly increase the blood pressure and does not involve Valsalva-type movements. This study helped obtain basic data that could be utilized for adapting the Tai Chi Yuttari-exercise for additional populations.

\section{ACKNOWLEDGEMENTS}

The authors thank the staff of the Health and Welfare Division, Kashiwara City Hall, who cooperated with this study. The authors also thank Prof. Seiji Yasumura from the Department of Public Health, Fukushima Medical University for supporting with this study.

\section{REFERENCES}

[1] Low, S., Ang, L.W., Goh, K.S. and Chew, S.K. (2009) A systematic review of the effectiveness of Tai Chi on fall 
reduction among the elderly. Archives of Gerontology and Geriatrics, 48, 325-331.

http://dx.doi.org/10.1016/j.archger.2008.02.018

[2] Blake, H. and Hawley, H. (2012) Effects of Tai Chi exercise on physical and psychological health of older people. Current Aging Science, 5, 19-27.

[3] Schleicher, M.M., Wedam, L. and Wu, G. (2012) Review of Tai Chi as an effective exercise on falls prevention in elderly. Research in Sports Medicine, 20, 37-58.

[4] Wu, G. and Millon, D. (2008) Joint kinetics during Tai Chi gait and normal walking gait in young and elderly Tai Chi Chuan practitioners. Clinical Biomechanics, 23, 787795. http://dx.doi.org/10.1016/j.clinbiomech.2008.02.001

[5] Nomura, T., Sugeno, N., Nagano, K., Takato, J., Ueki, S., Yanagi, H., Kikuchi, H. and Yasumura, S. (2007) A preliminary study on the effect of care prevention with development of a Tai Chi-like exercise. Journal of Rehabilitation and Health Sciences, 5, 1-6.

[6] Nomura, T., Nagano, K., Takato, J., Ueki, S., Matsuzaki, Y. and Yasumura, S. (2011) The development of a Tai Chi exercise regimen for the prevention of conditions requiring long-term care in Japan. Archives of Gerontology and Geriatrics, 52, e198-e203. http://dx.doi.org/10.1016/j.archger.2010.11.010

[7] Mori, K., Nomura, T., Akezaki, Y., Kataoka, S., Nakamata, E., Asada, F., Mori, S., Kai, S. and Watanabe, M. (2013) Dose three months of Tai Chi Yuttari-exercise improve the cardio-ankle vascular index? Randomized controlled trial. Research in Exercise Epidemiology, 52, 7180.

[8] Fujimoto, S., Yamazaki, S., Wakabayashi, A., Matsuzaki, Y. and Yasumura, S. (2011) The effects of Tai-chi exercise for the prevention of long-term care in community-

\section{APPENDIX}

\section{Abbreviation List}

AV-sitting: advanced version sitting-position pattern

AV-standing: advanced version standing-position pattern

BV-sitting: basic version sitting-position pattern

BV-standing: basic version standing-position pattern

BW: body weight

DBP: diastolic blood pressure dwelling frail elderly people. New care-need certification and mortality. Nippon Ronen Igakkai Zasshi, 48, 699-706. http://dx.doi.org/10.3143/geriatrics.48.699

[9] Kitakata City in Fukushima Prefecture and Yasumura, S. (2011) Tai Chi Yuttari-exercise for prevent the need for long-term care; revised edition with DVD. Iwaki Telework Center, Fukushima.

[10] Borg, G.A. (1982) Psychophysical bases of perceived exertion. Medicine \& Science in Sports \& Exercise, 14, $377-381$.

http://dx.doi.org/10.1249/00005768-198205000-00012

[11] Karvonen, M.J., Kentala, E. and Mustala, O. (1957) The effects of training on heart rate; a longitudinal study. Annales Medicinae Experimentalis et Biologiae Fenniae, 35, 307-315.

[12] Yoshida, M., Yamada, T. and Takeda, M. (2007) Evaluation of the intensities of exercise for elderly people to prevent worsening of disability. The Journal of Japan Academy of Health Sciences, 10, 73-79.

[13] Hasegawa, C., Kaneko, Y. and Yoshika, M. (2009) Original exercise by applying a regional dance; looking for a possibility of "SAKADO YOSAKOI-EXERCISE". Journal of Gymnastics for All, 6, 10-18.

[14] Japanese Ministry of Health, Labour and Welfare (2006) Exercise and physical activity guide for health promotion 2006.

http://www0.nih.go.jp/eiken/programs/pdf/exercise_guide .pdf

[15] Kakiyama, T., Yokoyama, N., Maeda, S., Kuno, S., Takaishi, M. and Matsuda, M. (2001) Effects of low-intensity exercise training for 6 months on arterial distensibility in middle-aged and elderly women. The Journal of Japanese Society of Clinical Sports Medicine, 9, 226-233.

DL: dyslipidemia

HR: heart rate

HT: hyper tension

METs: metabolic equivalents

MV: muscle volume

SBP: systolic blood pressure

SFV: somatic fat volume

$\mathrm{VO}_{2}$ : oxygen uptake

RQ: respiratory quotient

$\mathrm{RR}$ : respiration rate 